\title{
Les « promesses électorales » des bêtabloquants pour le choc septique seront-elles tenues?
}

\author{
Election Promises of Betablockers in Sepsis: Can we Really Count on It?
}

\author{
R. Favory · B. Lévy \\ (C) SRLF et Lavoisier SAS 2015
}

Le choc septique reste grevé d'une mortalité importante, même si la tendance est à la baisse depuis l'adoption des recommandations internationales de la Surviving Sepsis Campaign au début des années 2000. En parallèle, la plupart des thérapeutiques initialement prometteuses ont finalement échoué à améliorer la mortalité (protéine $\mathrm{C}$ activée, hydrocortisone) ou se sont révélées délétères (contrôle strict de la glycémie par insulinothérapie). Dans l'arsenal des thérapeutiques, il reste probablement la prise en charge précoce du choc septique (EGDT de l'étude de Rivers), qui est ancrée dans les pratiques des réanimateurs, y compris dans les groupes contrôles des deux récentes études multicentriques négatives nord-américaine (Process) et australienne (ARISE).

L'idée d'utiliser des bêtabloquants au cours des états hyperkinétiques septiques ne date pas d'hier [1]. Mais le « passage à l'acte » clinique est beaucoup plus récent. Ceci dit, si on met dans la balance les effets positifs et négatifs des catécholamines, on serait plutôt tenté de « décatécholaminiser » nos patients, du moins après la phase de sauvetage initiale. Augmenter le débit cardiaque afin d'augmenter le transport en $\mathrm{O}_{2}$ par l'utilisation d'agents inotropes positifs augmente la mortalité [2]. Facteurs de risque de choc postarrêt cardiaque, initiatrices des cardiomyopathies de stress, les catécholamines peuvent clairement avoir des effets délétères. Dans la cardiopathie septique - réversible en général en quelques jours -, on constate, quand des autopsies ont été réalisées, des anomalies histologiques compatibles avec une toxicité des catécholamines (bandes de contraction, infiltrats de cellules mononucléées...) [3]. La troponine augmente d'ailleurs souvent dans ce contexte, même si la macrocirculation coronaire s'adapte a priori à la situation. Chez la sou-

\footnotetext{
R. Favory $(\bowtie)$

Pôle de réanimation, hôpital Roger Salengro, rue Émile Laine, CHRU de Lille

e-mail : raphael.favory@chru-lille.fr

B. Lévy

Service de réanimation médicale, CHU Nancy Brabois, Institut du Cœur et des Vaisseaux, F-54500 Vandœuvre-lès-Nancy
}

ris, on retrouve les caractéristiques d'une hibernation myocardique : malgré une perfusion et une oxygénation préservées, on retrouve une dysfonction contractile, une augmentation de la captation de glucose et de l'expression de GLUT4, ainsi qu'une augmentation des dépôts de glycogène [4].

Il n'est donc pas logique de continuer à « fouetter» le système cardiovasculaire dès lors que la périphérie s'est mise en hibernation. Au contraire, diminuer l'état hyperkinétique pourrait avoir des effets intéressants, sur le myocarde bien sûr en permettant potentiellement une meilleure efficacité du remplissage diastolique et une diminution de la consommation en oxygène du myocarde, sur la toxicité des catécholamines circulantes sur le plan inflammatoire, perméabilité vasculaire, coagulation... [5]. Chez les patients brûlés, chez qui un état hyperkinétique est également présent, les bêtabloquants semblent prometteurs [6].

Concernant le sepsis, l'utilisation d'esmolol (bêtabloquant de demi-vie courte, cardiosélectif, sans activité sympathomimétique intrinsèque) lors de pneumonies infectieuses modérément sévères s'est révélée faisable [7]. Ensuite, l'étude pilote de Morelli sur 25 patients en état de choc septique a montré des résultats encourageants [8]. L'étude n'était pas contrôlée, rendant toute conclusion définitive difficile, mais toujours est-il que la mise en route d'esmolol intraveineux chez des patients stabilisés après 24 heures de prise en charge semblait bien tolérée. Les patients devaient avoir une pression artérielle moyenne de plus de $65 \mathrm{mmHg}$, une $\mathrm{SvO}_{2}$ supérieure à $65 \%$, une pression veineuse centrale (PVC) supérieure à 8 et une pression pulmonaire d'occlusion (PAPO) supérieure à $12 \mathrm{mmHg}$. L'objectif était de maintenir la fréquence cardiaque en-dessous de 95 battements par minute pendant 24 heures. En 24 heures avec ce traitement, l'index cardiaque diminuait et le volume d'éjection systolique augmentait parallèlement. Aucun patient n'était traité par inotrope positif. Sous ce traitement, le $\mathrm{pH}$ se normalisait et les doses de noradrénaline diminuaient. Le transport en $\mathrm{O}_{2}$ diminuait, mais la consommation en $\mathrm{O}_{2}$ également. La microcirculation appréciée par vidéomicroscopie au niveau 
sublingual s'améliorait également de façon notable et devenait moins hétérogène. Bien sûr, en l'absence de placebo, la part de l'évolution naturelle des patients n'était pas quantifiable.

L'étude suivante était comparative, randomisée en ouvert [9]. Les patients étaient inclus $24 \mathrm{~h}$ après le début du choc septique s'ils restaient tachycardes. L'esmolol en intraveineux était titré de manière à obtenir une fréquence cardiaque inférieure à 95 battements par minute et poursuivi jusqu'au décès ou à la sortie de réanimation. Le critère de jugement principal était la baisse de la fréquence cardiaque. Considérant ce critère principal, l'étude était positive. De plus, la faisabilité de ce traitement en termes de sécurité était confirmé, et ce d'autant que, parmi les critères de jugement secondaires, on constatait une baisse des doses nécessaires de noradrénaline, une amélioration du débit de filtration glomérulaire et une baisse de mortalité dans le groupe esmolol. Cette dernière donnée a fait couler beaucoup d'encre et de salive depuis la publication de l'article. En effet, la mortalité dans le groupe contrôle est très importante. Un des arguments avancés par les auteurs est que, dans leur registre précédent, les patients ayant une fréquence cardiaque encore élevée à 24 heures de réanimation avaient ce même taux de mortalité élevé (sous-groupe de patients possiblement très graves). Cette donnée trouve peut-être un début de réponse dans l'étude micro-SOAP, qui montre que, chez les patients tachycardes (fréquence cardiaque > 90) seulement, les altérations microcirculatoires sont indépendamment prédictives de mortalité. Il est évident que les résultats de Morelli, bien que très intéressants et finalement pas si surprenants (peutêtre même encore moins que dans l'insuffisance cardiaque il y a 20 ans, et pourtant...), sont à tempérer. Premièrement, il faut comprendre pourquoi les bêtabloquants ont fonctionné : effet purement cardiovasculaire, effet anti-inflammatoire, effet métabolique... Il n'est par ailleurs pas exclu que la poursuite de l'esmolol pendant toute la durée de séjour ait prévenu des événements cardiovasculaires chez les patients traités. Une étude récente nous montre que les patients survivants d'un choc septique (même si ça a été regardé davantage à distance que dans l'étude de Morelli) ont de facto un surrisque cardiovasculaire, en faisant peut-être de l'antécédent de choc septique un nouveau facteur de risque cardiovasculaire [10]...
Deuxièmement, il faut reproduire ces résultats dans d'autres centres, dans le cadre bien sûr d'études déclarées, hors traitement de troubles du rythme supraventriculaires, qui correspond à l'autorisation de mise sur le marché (AMM) en France.

Enfin, il faudra déterminer le cas échéant quels patients pourraient bénéficier de ce traitement, sous couvert fort probablement d'un monitorage rapproché.

Pour conclure, il existe un rationnel fort en faveur de l'utilisation des bétabloquants cardiosélectifs à demi-vie courte dans le choc septique. Afin de ne pas reproduire les erreurs passées, il est nécessaire d'identifier le sous-groupe de patients pouvant en bénéficier, ainsi que le bon timing d'initiation.

\section{Références}

1. Berk JL, Hagen JF, Beyer WH, et al (1969) The treatment of endotoxin shock by beta adrenergic blockade. Ann Surg 169:74-81

2. Gattinoni L, Brazzi L, Pelosi P, et al (1995) A trial of goaloriented hemodynamic therapy in critically ill patients. $\mathrm{SvO} 2$ Collaborative Group. N Engl J Med 333:1025-32

3. Schmittinger CA, Dunser MW, Torgersen C, et al (2013) Histologic pathologies of the myocardium in septic shock: a prospective observational study. Shock 39:329-35

4. Levy RJ, Piel DA, Acton PD, et al (2005) Evidence of myocardial hibernation in the septic heart. Crit Care Med 33:2752-6

5. Rudiger A (2010) Beta-block the septic heart. Crit Care Med 38 (10 Suppl):S608-12

6. Herndon DN, Hart DW, Wolf SE, et al (2001) Reversal of catabolism by beta-blockade after severe burns. $\mathrm{N}$ Engl J Med 345:1223-9

7. Gore DC, Wolfe RR (2006) Hemodynamic and metabolic effects of selective betal adrenergic blockade during sepsis. Surgery 139:686-94

8. Morelli A, Donati A, Ertmer C, et al (2013) Microvascular effects of heart rate control with esmolol in patients with septic shock: a pilot study. Crit Care Med 41:2162-8

9. Morelli A, Ertmer C, Westphal M, et al (2013) Effect of heart rate control with esmolol on hemodynamic and clinical outcomes in patients with septic shock: a randomized clinical trial. JAMA 310:1683-91

10. Yende S, Linde-Zwirble W, Mayr F, et al (2014) Risk of cardiovascular events in survivors of severe sepsis. Am J Respir Crit Care Med 189:1065-74 